\title{
Eclampsia: an overview clinical presentation, diagnosis and management
}

\section{Introduction}

\section{Preeclampsia and eclampsia}

Hypertensive disorders are among the most common medical complications of pregnancy, affecting about $7-10 \%$ of all pregnant women. These disorders are an important cause of maternal and perinatal morbidity. ${ }^{1,2}$ Preeclampsia is classically recognized as hypertension that begins after week 20 of pregnancy with significant proteinuria that disappears until 12weeks after the labor. The Australasian Society for the Study of Hypertension in Pregnancy (ASSHP) already had suggested that proteinuria not be included as a diagnostic criterion for preeclampsia. ${ }^{3}$ According to The American Congress of OBGYN Task Force on Hypertension in Pregnancy, proteinuria was eliminated as a requirement for the diagnosis of preeclampsia. ${ }^{4}$ HELLP syndrome represents a severe form of preeclampsia-eclampsia, currently regarded as a variant of severe preeclampsia or a complication of the disease, and is characterized by hemolysis, elevated liver enzymes and low platelets. ${ }^{5}$ HELLP syndrome and eclampsia seem to share the component of endothelial dysfunction in their pathophysiology. The incidence of HELLP syndrome in association with eclampsia is 10.8$32.1 \%,{ }^{6,7}$ and the incidence of eclampsia in association with HELLP syndrome is $6-52 \%{ }^{8,9}$

The usual clinic practice in many prenatal clinics is to initiate urine dipstick protein measurements at each prenatal visit after 20 weeks of pregnancy. Gestational proteinuria is defined as urinary protein excretion of at least $300 \mathrm{mg}$ per 24-hour timed collection or persistent proteinuria $(1+$ on dipstick on at least two occasions at least 4hours apart). In some women hypertension or proteinuria may be absent in 10 to $15 \%$ of women who develop HELLP syndrome and in $20-25 \%$ of those who develop eclampsia, defaulting the diagnosis. ${ }^{10,11}$ Substantial proteinuria ( $3+$ in dipstick) is present in $48 \%$ of the cases of eclampsia but absent in another $14 \%$ of cases. ${ }^{12}$ Eclamptic seizures can develop before proteinuria is identified, and proteinuria may fluctuate over any 24-hour period.

A large proportion of women with eclampsia do not exhibit antecedent hypertension. It had been suggested that eclamptic women without significant hypertension had even lower non pregnant blood pressure, resulting in a lower limit of the cerebral autoregulatorycurve. ${ }^{13}$ In addition, circulating factors capable of initiating blood-brain barrier disruption may be involved, increasing the susceptibility to eclampsia. ${ }^{14,15}$ Prevention or control of convulsive episodes is very important in patients with severe preeclampsia. Eclampsia is related to adverse outcomes including cerebral hemorrhage, stroke, seizures, cardiopulmonary compromise, renal failure, liver hematoma or rupture, placental abruption, preterm delivery and death. ${ }^{10}$

\section{Eclampsia diagnosis}

Eclampsia is defined as the occurrence of grand mal seizures during pregnancy or during/after delivery in a woman with preeclampsia, not attributable to other causes. Almost all cases occur in the third trimester $(91 \%)$, after 28 weeks of pregnancy. ${ }^{12}$ Preeclampsia or eclampsia occurring before 20 weeks of pregnancy can occur in
Volume 3 Issue 2 - 2016

\author{
Rose Gasnier \\ Department of Obstetrics and Gynecology, Universidade \\ Federal do Rio Grande do Sul, Brasil
}

Correspondence: Rose Gasnier, Hospital de Clínicas de Porto Alegre, Universidade Federal do Rio Grande do Sul, Brasil, Email gasnier.rose@gmail.com

Received:September 25, 2015 | Published: November 28, 2016

cases of molar or hydropic degeneration of placenta with or without a coexistent fetus. ${ }^{16}$ Convulsions in the first half of pregnancy in association with hypertension and proteinuria should be initially considered as eclampsia, and another pathologic process should be excluded. The incidence of eclampsia varies between $0.2-0.5$ percent of all deliveries according to the 1988 World Health Organization's International Collaborative Study of Hypertensive Disorders of Pregnancy. ${ }^{6}$ The reported incidence is usually much higher in tertiary referral medical centers and in patients that do not obtain prenatal care. A global decrease in the incidence of eclampsia is supported by studies in the United States, United Kingdom and Canada. The rate is 30times higher in developing countries. ${ }^{17}$ Patients with ongoing seizures should be given anticonvulsant therapy when magnesium sulfate is insufficient to control them. These patients rarely go on to develop epilepsy. A rapid increase in blood pressure followed by convulsions is usually preceded by syntoms as a headache or visual disturbances. ${ }^{11}$ Several clinical symptoms are indicative of eclampsia diagnosis: persistent occipital or frontal headaches, blurred vision, photophobia, epigastric or right upper quadrant pain, and altered mental status. In most cases can be seen at least one of these symptoms in $59-75 \%$ of the cases. ${ }^{11}$ Epigastric or right upper pain precedes hepatic infarction and hemorrhage or catastrophic rupture of a subcapsular hematoma. A population-based survey on eclampsia - BEST survey (British Eclampsia Survey Team) in the UK, reported that 50\% of women with eclampsia had headache preceding convulsions, 19\% experienced visual disturbances and 19\% complained of epigastric pain. ${ }^{18}$ Antepartum and preterm eclampsia were found to be more likely to be preceded by prodromal symptoms.

\section{Maternal morbidity and mortality \\ HELLP syndrome}

Eclampsia is one of the most important reasons for maternal mortality in high and low-income countries. According to a review of the literature about maternal mortality, concurrent HELLP syndrome and eclampsia was the principal cause or contributing condition for eclampsia-related mortality in low and high-income countries. ${ }^{19}$ The deaths probably are related to HELLP syndrome rather than seizures 
alone, and the control or absence of control of seizures did not appear to affect the cause or timing of death. The majority of maternal deaths were due to intracerebral hemorrhage, data that is classically known in cases of eclampsia and in concordance to most reports. ${ }^{20}$ Maternal mortality from hypertensive disorders of pregnancy seems to be associated with the triad of seizures, severe systolic hypertension and thrombocytopenia secondary to HELLP syndrome. ${ }^{21}$ Comparison of two eras of eclampsia data from the University of TennesseeMemphis, HELLP syndrome was more prevalent in patients with early compared to late eclampsia. ${ }^{22}$ The incidence of eclampsia during the second era was nearly half of that reported for the first era at the same institution. The most common maternal complication in eclampsia cases was recurrent seizures followed by persistent neurologic deficits. The reduction in eclampsia cases likely is due to the routine use of magnesium sulfate following recommendations from the MAGPIE Study ${ }^{23}$ Systolic and diastolic blood pressures were higher in the antepartum/intrapartum eclampsia patient group compared to postpartum eclampsia. The earlier that eclampsia occurred during gestation, the greater the disease severity and the more likely clinical presentation composed of hypertension, proteinuria and evidence of HELLP syndrome.

Investigators at the University of Mississippi, reviewing three decades of patients with HELLP syndrome, found no evidence of significant worsening of maternal or perinatal outcomes when both conditions occurred together, a finding different from most other series. They concluded that eclampsia does not appear to contribute a significant adverse impact upon the course or outcome of HELLP syndrome pregnancies when glucocorticoids are a routinely used component of HELLP management. ${ }^{24}$ To reduce the number of deaths from eclampsia, early diagnosis and management of HELLP syndrome including timely delivery are highly desirable, including prevention of seizure and severe systolic hypertension. The prevention of occurrence of multiple seizures seems important because most women that suffer multiple seizures with eclampsia have evidence of cerebral infarction and HELLP syndrome. ${ }^{25}$

Expectant management of severe preterm preeclampsia is controversial in view of maternal risks with possible complications of placental abruption, eclampsia, consumptive coagulopathy, renal failure, hypertensive encephalopathy, and ruptured hepatic hematoma. Among women with partial HELLP syndrome, as well as those with severe forms of preeclampsia, infant outcomes are related to gestational age at delivery. ${ }^{26}$ Approximately $16 \%$ of cases of HELLP syndrome occur in concert with eclampsia and $10 \%$ of eclampsia cases occur in patients with HELLP syndrome. ${ }^{27}$ About 17 deaths each year are associated with eclampsia and concurrent HELLP syndrome in the USA. ${ }^{19}$

\section{Maternal mortality}

Hemorrhagic stroke is an important cause of pregnancy-related mortality ${ }^{28}$ In a large proportion of cases, the cause is unclear, often related to preeclampsia-eclampsia. ${ }^{29}$ The rates of preeclampsia reported in patients with intracerebral hemorrhage (ICH) in pregnancy have ranged from 14 to $50 \% .{ }^{30}$ Cerebral hemorrhages are more likely in older women with chronic hypertension. Infrequently the cause of ICH is a ruptured berry aneurysm or arteriovenousmal formation, part of differential diagnosis. ${ }^{11}$ Eclampsia is associated with a risk of $0-1.8 \%$ for maternal death in developed countries but the maternal mortality rate may be as high as $14 \%$ in developing countries. ${ }^{14}$
Each year approximately 63,000 women worldwide die of eclampsia and preeclampsia, and $99 \%$ of these deaths occur in low-income countries. ${ }^{31}$ Multiple seizures before medical attention and poor prenatal care are related to the high maternal mortality reported from developing countries. Perinatal mortality and morbidity are high in eclamptic pregnancies, with reported perinatal death from 5.6-11.8\%, related to prematurity, abruption placentae, and severe fetal grown restriction. The rate of preterm delivery is $50 \%$, with about $25 \%$ of the cases occurring before 32 weeks of pregnancy. ${ }^{14}$

The pathophysiologic changes that occur within the cerebrovascular system in the setting of preeclampsia and eclampsia predispose pregnant women to ischemic and hemorrhagic stroke. Understanding the underlying mechanisms helps prevent acute vascular events before delivery and in the postpartum period..$^{32}$ In a single year observational study that has carried out in Bangladesh, there were 4727 births among which were 124 (2.62\%) women with eclampsia. ${ }^{33}$ Most of the patients were young, in a first pregnancy with antepartum eclampsia; more than half were term gestations with infrequent or absent prenatal care. All patients had convulsions before admission and $85 \%$ were unconscious. Complications of eclampsia were found in 30\% of the cases: pulmonary edema, postpartum hemorrhage, cerebral vascular accident, HELLP syndrome, disseminated intravascular coagulation, acute renal failure. Criteria-based audits (CBA) have been used to improve clinical management in developed countries, and recently have been introduced in the developing world. A CBA of eclampsia cases was conducted in a hospital in Tanzania, Africa. ${ }^{34}$ Management practices were evaluated using evidence-based criteria for appropriate care. One year after initiation of the audit, some improvements noted included cesarean section undertaken within 2hours of decision (33 vs. $66 \%$ ), proper use of partogram (75 vs. $95 \%$ ), urine for albumin test (61 vs. $99 \%$ ), treatment with steroids for pulmonary maturity (2 vs. $24 \%$ ), full blood count ( 28 vs. $93 \%$ ), serum urea and creatinine (44 vs. $86 \%$ ), and laboratory testing of liver enzymes (4 vs. $86 \%$ ). There was a significant reduction of maternal deaths (7.7 vs. $0 \%)$.

\section{Treatment-magnesium sulfate}

The recommended regimen of magnesium sulfate is a loading dose of 4-6g given over 20minutes, followed by a maintenance dose of $1-2 \mathrm{~g}$ per hour as a continuous intravenous solution. Magnesium sulfate is initiated at the beginning of the observation period and then continued during labor and for at least 24 hours postpartum. ${ }^{35-37}$ Patients with eclampsia should receive postpartum magnesium sulfate for up to 48hours according to some authors. In those with abnormal renal function, the dose should be reduced and magnesium levels monitored. About $10 \%$ of eclamptic women have a second convulsion after starting treatment, and another bolus of $2 \mathrm{~g}$ can be given intravenously over 3 to 5 minutes. Recurrent seizures can be treated with sodium amobarbital, $250 \mathrm{mg}$ intravenously over 3 to 5 minutes. ${ }^{38}$

Magnesium sulfate is the drug of choice to prevent occurrence and recurrence of convulsions in women with preeclampsia. The results of randomized trials revealed that is superior to placebo or no treatment, and also to other anticonvulsants, for prevention of convulsions in women with severe preeclampsia. These trials revealed that magnesium sulfate was associated with a significantly lower rate of recurrent seizures $(9.4 \%$ versus $23.1 \%$, RR $0.4195 \%$ CI $0.32-0.51)$ and a lower rate of maternal death (3\% versus $4.8 \%$ RR $0.6295 \%$ CI 0.39-0.99) than that observed with other agents. Based on a number of studies, magnesium most likely exerts a specific anticonvulsant 
effect on the cerebral cortex, and the woman stops convulsing after the initial administration. After an hour or two, regains consciousness sufficiently to be oriented, a rapid recovery that may not occur with other drugs. Parenteral administration of magnesium sulfate is cleared almost totally by renal excretion, and magnesium toxicity is avoided by ensuring that urine output is adequate, the patellar or biceps reflex is present, and there is no respiratory depression. Neonatal depression occurs only if there is severe hypermagnesemia at delivery. Fetal heart beat-to-beat variability can be reduced. A protective effect of magnesium against cerebral palsy in very-low-birth weight infants has been shown and has been used in cases of imminent labor prior to 32 weeks of pregnancy.

The multinational Eclampsia Trial Collaborative Group Study was funded in part by the World Health Organization and coordinated by the National Perinatal Epidemiology Unit. Women allocated to magnesium sulfate therapy had a $50 \%$ reduction in recurrent seizures compared with those given diazepam, and a $67 \%$ reduction compared to phenytoin. Maternal deaths were reduced in women given magnesium sulfate compared to diazepam and phenytoin. ${ }^{39}$ During the Magpie Trial Collaborative Trial (Magnesium Sulfate for Prevention of Eclampsia), women allocated to receive magnesium sulfate had 58\% lower risk of eclampsia than those given placebo. The overall number of patients treated to prevent one case of eclampsia was 91. For women with severe preeclampsia, this number was 63 , and for those with mild preeclampsia were 109. The risk of placental abruption was also significantly lower in the magnesium sulfate group..$^{23}$

In the Dallas, Texas Parkland Hospital experience, preeclampsia management was changed since 2000. Prior to the change in practice, the eclampsia rate was $8.7 / 10,000$. During the following decade using a new protocol, the rate of eclampsia rate increased to $14 / 10,000$ without any deaths from eclampsia. Since 2000, the use of magnesium sulfate has been restricted for use only in women with severe gestational hypertension $(\mathrm{BP} \geq 160 / 110 \mathrm{mmHg}$ ) or less severe hypertension $(\geq 140 / 90)$ accompanied by headache, epigastric pain, proteinuria at least $2+$, thrombocytopenia, or elevated creatinine or transaminase levels, without another substantive protocol changes. Such observations suggest that a return to more liberal use of magnesium sulfate for preeclampsia prophylaxis might lower rates of eclampsia. ${ }^{40}$ In some comparisons of the Collaborative Eclampsia Trial, women allocated to magnesium sulfate therapy were less likely to be artificially ventilated, to develop pneumonia, and to be admitted to intensive care unit than those given phenytoin. Neonates from women given magnesium sulfate were significantly less likely to require intubation at delivery and to be admitted to the neonatal intensive care unit compared with infants whose mothers received phenytoin. ${ }^{39}$

\section{Eclampsia in developing countries}

Poor prenatal care is associated with a large number of deaths caused by eclampsia in developing countries. The low incidence of eclampsia in developed countries is probably related to prevention of eclampsia cases in women with a classic preeclampsia presentation and progression to develop severe features of preeclampsia. Most eclamptic cases described in reported series from the United Stated and Europe have an atypical presentation (abrupt onset, development of convulsions while receiving prophylactic magnesium sulfate, or onset of convulsions beyond 48 hours after delivery). ${ }^{17}$ Most cases occur in hospitalized women, not preceded by warning signs or symptoms. Recent data from large series of eclamptic women from the United States and Europe indicate that about $20-40 \%$ of eclamptic women do not have any premonitory signs or symptoms before seizure onset. ${ }^{11}$ In many of these cases, the onset of the seizure is abrupt with the course of the disease not having followed from mild to severe before the onset of eclampsia.

Eclampsia is more common among adolescents, and around $85 \%$ of all births among adolescent mothers occur in low-income countries. A study from Japan showed that the risk of eclampsia declines by $3 \%$ for every year increase in maternal age. ${ }^{41}$ Half of eclampsia cases occur under 20years, and half of the cases of death seem to occur in this age group. Eclampsia without HELLP syndrome is common in the Latin America countries but has a good prognosis. The decline in the incidence of eclampsia in Latin America might be explained by an increased use of magnesium sulfate in women with severe hypertensive disorders of pregnancy, especially after the MAGPIE trial. Sometimes the rise in blood pressure and presence of proteinuria are not significant, but the continuation of the pregnancy can be very dangerous with evolution to HELLP syndrome with DIC, new episodes of convulsions and sometimes ICH. Besides, in developing countries without routine laboratory testing of patients prior to eclampsia, the diagnosis of HELLP syndrome may occur later during care.

\section{Eclampsia -clinical management}

Supportive care should be given to prevent serious maternal injury and aspiration, assess and establish airway patency, ensure maternal oxygenation, and start immediately magnesium sulfate according to the protocols. The next step in the management of eclampsia is to reduce blood pressure. The objectives are to avoid loss of cerebral autoregulation and to prevent congestive heart failure without compromising cerebral perfusion or uteroplacental flow. The goal is considered systolic BP between 140 and $160 \mathrm{~mm} \mathrm{Hg}$ and diastolic BP between 90 and $105 \mathrm{~mm} \mathrm{Hg}$. This can be achieved with bolus 5 to $10 \mathrm{mg}$ doses of hydralazine or labetalol (20 to $40 \mathrm{mg}$ intravenously) every 15 minutes as needed. Delivery of the fetus is definitive treatment for eclampsia independently of the gestational age, although it is important to note that manifestations of PRES (posterior reversible encephalopathy syndrome) may arise only after delivery. ${ }^{11}$

Maternal and fetal conditions are assessed, and a decision is made regarding the need for delivery. Those with a gestational age of 24 to 34 weeks are given corticosteroids to accelerate fetal lung maturity. Maternal evaluation includes monitoring of blood pressure, urine output, cerebral status, and the presence of epigastric pain, tenderness, labor or vaginal bleeding. Laboratory evaluation includes a platelet count, liver enzymes, and serum creatinine. Fetal evaluation includes continuous fetal heart monitoring, a biophysical profile status, and ultrasonography assessment of fetal growth with Doppler evaluation and amniotic fluid. Patients with resistant severe hypertension despite or persistent cerebral symptoms while on magnesium sulfate are delivered irrespective of fetal gestational age. The objective of treating acute severe hypertension is to prevent cerebrovascular and cardiovascular complications such as encephalopathy, seizures, intracranial hemorrhage, and congestive heart failure. ${ }^{13}$

Maternal hypoxemia and hypercarbia cause fetal heart rate and uterine activity changes during and immediately after an eclamptic seizure, related to reduced uterine blood flow. The fetal heart 
rate tracing may reveal bradycardia, transient late decelerations, decreased beat-to-beat variability, and compensatory tachycardia. These changes usually resolve spontaneously within 3 to 10 minutes after the termination of the seizure and the correction of maternal hypoxemia-this is not the time to emergently deliver the fetus. It may take longer for the fetal heart rate pattern to return to baseline in an eclamptic woman whose preterm fetus is growth restricted. Placental abruption can occur after the seizure and should be considered if uterine hyperactivity remainsor fetal bradycardia persists. In antepartum eclampsia, labor may begin shortly after a convulsion and progress rapidly. ${ }^{11}$ The optimal time interval between seizure and delivery has not been clarified. It is known that resuscitation of the fetus in utero may be better for the fetus unless there is post-seizure persistent non-reassuring fetal status. Classically the mainstay of the eclampsia treatment has always been the delivery of the patient, and some authors consider eclampsia to be an absolute contraindication to expectant management. Concerns about delay of delivery include disease progression to multiorgan disease, maternal central nervous system and placental abruption..$^{42-43}$

It is desirable to initiate corticosteroids for fetal lung maturation especially in very preterm pregnancy, but it is not deemed necessary to postpone delivery in the presence of indication to immediate delivery. A course of corticosteroids appears to offer notable fetal pulmonary benefit without significantly increasing maternal or fetal risks. In addition, it is possible to discern additional maternal effects from a corticosteroid course such as possibly faster recovery from the brain effects of PRES with faster sensorium normalization, possible reduction of risk to see HELLP syndrome develop or progress, improvements in maternal platelet count to enable regional anesthesia, and enhancing the chance of vaginal delivery. ${ }^{44}$ Corticosteroids have been used for treating women with HELLP before and after delivery, but their clinical use is still a subject of debate. Despite of this, when aggressive corticosteroids are not used, significant maternal morbidity or mortality can occur that might otherwise have been avoided. ${ }^{45}$ Case reports of mortality and morbidity with cerebral hemorrhage and stroke were not treated with corticosteroids.

If undergoing cesarean delivery, the Task Force recommends intraoperative administration of parenteral magnesium sulfate. The presence of eclampsia is not an indication for cesarean delivery. The decision to perform a cesarean delivery should be based on gestational age, fetal condition, presence of labor, and cervical Bishop Score. Regional anesthesia is contraindicated in the presence of coagulopathy or severe thrombocytopenia (platelet count $<50,000$ / $\mathrm{uL}$ ). In women with eclampsia, general anesthesia increases the risk for aspiration and failed intubation due to airway edema and is associated with marked increases in systemic and cerebral pressures during intubation and extubation, as well as elevated risk for aspiration pneumonia during the post-operative period. Thus general anesthesia is not recommended for use in the eclampsia patient if it can be avoided. ${ }^{11}$ Following delivery, women with eclampsia should receive close monitoring of vital signs, fluid intake and output, and symptoms for at least 48 hours. Intravenous magnesium sulfate is generally continued for 24-48hours, and antihypertensive drugs are employed if the systolic BP is at least $155 \mathrm{~mm} \mathrm{Hg}$ or if the diastolic $\mathrm{BP}$ is at least $105 \mathrm{~mm} \mathrm{Hg.}{ }^{11}$ During the postpartum period there is mobilization of extracellular fluid, with increased risk for pulmonary edema and severe hypertension postpartum, especially in those cases with placental abruption, abnormal renal function and those with preexisting chronic hypertension. ${ }^{11}$

\section{Postpartum eclampsia}

About $5 \%$ of women with preeclampsia first manifest signs and symptoms after delivery ${ }^{46}$ and a third of eclamptic patients have their first seizure after delivery has occurred. ${ }^{47}$ The reported frequency of antepartum convulsion among recent series has ranged from $38-53 \%$, and the frequency postpartum ranges from 11 to $44 \% .^{12}$ Although most cases of postpartum eclampsia occur within the first 48hours, some cases can develop beyond 48hours postpartum and have been reported as late as 23 days postpartum. In the latter cases, an extensive neurologic evaluation is necessary. ${ }^{11}$ Latepostpartum eclampsia is defined as eclampsia that occurs more than 48 hours but less than 4 weeks after delivery. ${ }^{11}$ Some of these women demonstrate preeclampsia during labor or immediately postpartum (56\%), whereas others reveal evidence of the disease for the first time more than 48hours after delivery $(44 \%) .{ }^{17}$ Late postpartum eclampsia develops despite the use of prophylactic magnesium sulfate during labor and for at least 24 hours postpartum in women previously diagnosed with preeclampsia.

Patients typically develop manifestations of cerebral edema, including headache, nausea and vomiting, and cortical visual phenomena consistent with occipital lobe dysfunction before a seizure occurs. ${ }^{48}$ On the other side, eclampsia can manifest without warning or signs of preeclampsia in up to $40 \%$ of cases ${ }^{49,50}$ Classically, the delivery of the fetus and placenta resolves the clinical manifestations of preeclampsia, but in some patients the disease process can worsen after delivery. There is little information on how best to manage postpartum severe preeclampsia or postpartum eclampsia diagnosed for the first time after delivery. As mentioned previously, postpartum eclampsia can develop in the absence of hypertension and/or proteinuria. For women with severe preeclampsia diagnosed during the postpartum period or diagnosed before delivery but without treatment, the MAGPIE trial randomized 1335 patients after delivery (subgroup analysis) to receive either magnesium sulfate or placebo. There were no statistical differences between groups. The risk for eclampsia was very small (1\%) but was not reduced with the use of magnesium sulfate. Without statistical power to derive a definitive conclusion on this issue, there is no compelling evidence to support the use of magnesium sulfate for the prevention of eclampsia in these circumstances. In the group of patients whose eclampsia was diagnosed during the postpartum period, a subgroup analysis within the Collaborative Eclampsia Trial was undertaken: 419 postpartum eclampsia patients were randomized to magnesium sulfate versus diazepam or magnesium sulfate versus phenytoin. The recurrent convulsion rate was as follows: magnesium sulfate $10.9 \%$ versus diazepam $29.9 \%$, magnesium sulfate $6.3 \%$ versus phenytoin $14.7 \%$. This difference also was no significant. Of note is that even with the use of magnesium sulfate, the recurrent risk for convulsion was in the order of $6-11 \% .^{51}$

\section{The pres syndrome}

Severe cerebral vasogenic edema in PRES may cause compression of cerebral tissue, resulting in reduced perfusion, followed by ischemia, hypoxia, cytotoxic edema, and cell death. This may appear as cerebral white lesions or infarction on MRI several years later. Tonic-clonic seizures are not mandatory for the diagnosis of PRES and preeclamptic women may demonstrate signs, symptoms and imaging findings of PRES in the absence of an eclamptic seizure, especially in cases of early-onset preeclampsia. ${ }^{52}$ In addition, recent studies have 
documented cytotoxic cerebral edema in up to $25 \%$ of women who experienced eclamptic seizures. ${ }^{53}$ There is now evidence that women who have eclampsia may develop some degree of neurocognitive dysfunction, related in part to the total number of seizures. The affirmation by some authors in the past that treatment is optional has no current value. The reversibility of PRES and eclampsia has been questioned. ${ }^{54}$ As the edema progresses, oncotic and hydrostatic forces may lead to intraparenchymal hemorrhage and vasospasm with ischemic infarction. Seizures mark the late stage of disease and develop secondary to cerebral edema and breakdown of the bloodbrain barrier with disruption of the normal ionic gradients. The finding that a substantial number of eclampticpatients exhibit persistent neurocognitive deficits and white matter lesions located in the frontal lobe, like white matter lesions in elderly individuals related to dementia and cognitive decline as result of vascular disease, suggests an additional etiology other than ischemia from vasogenic edema. ${ }^{55}$ Of the women that develop eclampsia, $95 \%$ also develop PRES. ${ }^{56}$ The incidence of cerebral hemorrhage in patients who develop PRES is estimated to be between 10 and $15 \% .{ }^{57}$ The actual incidence of PRES in pregnancy may be much higher than initially recognized.

It is not clear whether the pathologic features in eclampsia are a cause or an effect of the convulsions. ${ }^{11}$ Autoregulation of the cerebral circulation is a mechanism for the maintenance of constant cerebral blood flow during changes in BP and may be altered in eclampsia. Currently it thought that eclampsia is probably the most common condition underlying the PRES syndrome. ${ }^{11}$ In the presence of endothelial dysfunction, sudden, even minute, elevations in systemic blood pressure may result in failure of autoregulation. Such areas of poorly perfused brain may ultimately be at risk for ischemia and infarction, all of which may give rise to the development of brain white matter lesions. Although eclamptic patients may manifest a variety of neurologic abnormalities, most have no permanent neurological deficits. Visual disturbances are relatively common during the acute phase of eclampsia, including transient cortical blindness, scotomata, visual neglect, and blurred vision, and can often be attributed to the presence of cerebral edema which likely is an expression of PRES syndrome. These neurologic abnormalities are probably due to a transient insult, such as hypoxia, ischemia, or edema.

Cerebral imaging is not necessary for the diagnosis and management of most women with eclampsia, but is indicated for patients with focal neurological deficits or prolonged coma. In these patients, hemorrhage and other abnormalities requiring specific pharmacological therapy or surgery must be excluded. Cerebral imaging also is helpful in patients who have an atypical presentation for eclampsia (onset before 20weeks of gestation or more than 48 hours after delivery, and eclampsia refractory to adequate magnesium sulfate therapy). ${ }^{11}$ Differential diagnosis is important in those cases. Other causes of seizures during pregnancy include a bleeding arteriovenous malformation, idiopathic seizure disorder and ruptured aneurysm.

\section{Acknowledgements}

None.

\section{Conflict of interest}

The author declares no conflict of interest.

\section{References}

1. Lindheimer MD, Umans JG. Explaining and predicting preeclampsia. N Engl J Med. 2006;335(10):1056-1058.

2. Sibai B, Dekker G, Kupferminc M. Pre-eclampsia. Lancet. 2005;365(9461):785-799.

3. Brown MA, Hague WM, Higgins J, et al. The detection, investigation and management of hypertension in pregnancy. Aust NZ J Obstet Gynaecol. 2000;40(2):133-1355.

4. Moussa HN, Arian SE, Sibai BM. Management of hypertensive disorders in pregnancy. Women's Wealth. 2014;10(4):385-404.

5. Haram K, Svendsen E, Abildgaard U. The HELLP syndrome: clinical issues and management. A review. BMC Pregnancy Childbirth. 2009;9:8.

6. Mattar F, Sibai B. Eclampsia. VIII. Risk factors for maternal morbidity. Am J Obstet Gynecol. 2000;182(2):307-312.

7. Martin JN, Rinehart BK, May WL, et al. The spectrum of severe pre-eclampsia: Comparative analysis by HELLP (hemolysis, elevated liver enzyme levels, and low platelet count) syndrome classification. Am J Obstet Gynecol. 1999;180(6 pt 1):1373-1384.

8. Haddad B, Baton JR, Livingston JC, et al. Risk factors for adverse maternal outcomes among women with HELLP (hemolysis, elevated liver enzymes, and low platelet count) syndrome. Am J Obstet Gynecol. 2010;183(2):444-448.

9. Cavkaytar S, Ugurlu EN, Karaer A, et al. Are clinical symptoms more predictive than laboratory parameters for adverse maternal outcome in HELLP syndrome? Acta Obstet Gynecol Scand. 2007;86(6):648-651.

10. Sibai BM. Diagnosis, controversies, and management of HELLP syndrome. Obstet Gynecol. 2004;103(5 Pt 1):981-999.

11. Sibai BM. Diagnosis, differential diagnosis and management of eclampsia. Obstet Gynecol. 2005;105:402.

12. Sibai BM, Hauth J, Caritis S, et al. Hypertensive disorders in twin versus singleton gestations. National Institute of Child Health and Human Development Network of Maternal-Fetal Medicine Units. Am J Obstet Gynecol. 2000;182(4):938-942.

13. Schreurs MP, Cipolla MJ, Al-Nasiry S, et al. Formerly eclamptic women have lower nonpregnant blood pressure compared with formerly pre-eclamptic women: a retrospective cohort study. BJOG. 2015;122(10):1403-1409.

14. Schreurs MP, Hubel CA, Bernstein IM, et al. Increased oxidized low-density lipoprotein causes blood-brain barrier disruption in early-onset preeclampsia through Iox-1. FASEB J. 2013;27(3):1254-1263.

15. Amburgey OA, Chapman AC, May V, et al. Plasma from preeclamptic women increases blood-brain barrier permeability: role of vascular endothelial growth factor signaling. Hypertension. 2010;56(5):10031008 .

16. Duley L. The global impact of pre-eclampsia and eclampsia. Sem Perinatol. 2009;33(3):130-137.

17. Sibai BM, Stella SC. Diagnosis and management of atypical preeclampsia-eclampsia. Am J Obstet. 2009;200(5):481.e1-481.e7.

18. Douglas KA. British Eclampsia Survey Team. Qual Health Care. 1992;1(2):142.

19. Vigil-De Gracia P. Maternal deaths due to eclampsia and HELLP syndrome. Int J Gynaecol Obstet. 2009;104(2):90-94. 
20. Lewis G. The confidential enquiry into maternal and child health (CEMACH). Saving mothers' Lives: reviewing maternal deaths to make motherhood safer 2003-2005. The seventh report on confidential enquires into maternal deaths in the United Kingdom. Obstet Med. 2008;1(1):54.

21. Vigil-De Gracia P, Garcia-Caceres E. Thrombocytopenia, hypertension and seizures in eclampsia. Int J Gynaecol Obstet. 1998;61(1):15-20.

22. Schenone MH, Miller D, Samson JE, et al. Eclampsia characteristics and outcomes: a comparison of two eras. J Pregnancy. 2013;2013:826045

23. Magpie Trial Collaborative Group. Do women with preeclampsia, and their babies, benefit from magnesium sulphate? The Magpie Trial: a randomized placebo-controlled trial. Lancet. 2002;359(9321):18771890

24. Keiser SD, Owens MY, Parrish MR, et al. HELLP syndrome with and without eclampsia. Am J Perinatol. 2011;28(3):187-194.

25. Zeeman GG, Fleckenstein JL, Twickler DM, et al. Cerebral infarction in eclampsia. Am J Obstet Gynecol. 2004;190(3):714-720.

26. Sibai BM. Evaluation and management of severe preeclampsia before 34 weeks gestation. Am J Obstet Gynecol. 2011;205(3):191-198.

27. Andersgaard AB, Herbst A, Johansen M, et al. Eclampsia in Scandinavia: incidence, substandard care, and potentially preventable cases. Acta Obstet Gynecol Scand. 2006;85(8):929-936.

28. Kittner SJ, Stern BJ, Feeser BR, et al. Pregnancy and the risk of stroke. N Engl J Med. 1996;335(11):768-774.

29. Sharshar T, Lamy c, Mas JL. Incidence and causes of stroke associated with pregnancy and puerperium: a study in public hospitals of Ile de France. Stroke in pregnancy Study Group. Stroke. 1995;26(6):930-936.

30. Yoshimatsu J, Ikeda T, Katsuragi S, et al. Factors contributing to mortality and morbidity in pregnancy-associated intracerebral hemorrhage in Japan. J Obstet Gynaecol Res. 2014;40(5):1267-1273.

31. Langer A, Villar J, Tell K, et al. Reducing eclampsia-related deaths - a call to action. Lancet. 2008;371(9614):705-706.

32. Razmara A, Bakhadirov K, Batra A, et al. Cerebrovascular complications of pregnancy and the postpartum period. Curr Cardiol Rep. 2014;16(10):532.

33. Parna FH, Latif T, Sultana N, et al. Maternal \& fetal outcome of eclamptic patients admitted in obstetrics \&gynaecology department of secondary care hospital in Bangladesh. Mymensingh Med J. 2013;22(3):522-526.

34. Kidanto HL, Wangwe $\mathrm{P}$, Kilewo $\mathrm{CD}$, et al. Improved quality of management of eclampsia patients through criteria based audit at Muhimbili National Hospital, Dar es Salaam, Tanzania. Bridging the quality gap. BMC Pregnancy and Childbirth. 2012;12:134.

35. Report of the National High Blood Pressure Education Program: Working Group report on high blood pressure in pregnancy. Am J Obstet Gynecol. 2000;183(1):S1-22.

36. Sibai BM. Diagnosis and management of gestational hypertension and preeclampsia. Obstet Gynecol. 2003;102(1):181-192.

37. ACOG Committee on Practice bulletin. Diagnosis and management of preeclampsia and eclampsia. Obstet Gynecol. 2002;99(1):159-167.
38. ACOG committee on practice bulletin. Obstet Gynecol. 2013;122:1122.

39. Which anticonvulsant for women with eclampsia? Evidence from the collaborative Eclampsia Trial. Lancet. 1995;346(8969):1455-1463.

40. Lindheimer MD, Cunningham FG. Avoiding the eclamptic convulsion. BJOG. 2014;121(12):1529.

41. Morikawa M, Cho K, Yamada T, et al. Risk factors for eclampsia in Japan between 2005 and 2009. Int J Gynaecol Obstet. 2012;117(1):6668.

42. Leitch CR, Cameron AD, Walker JJ. The changing patterns of eclampsia over a 60-year period. Br J Obstet Gynaecol. 1997;104(8):917-922.

43. Andersen WA, Harbert GM. Conservative management of pre-eclamptic and eclamptic patients: a re-evaluation. Am J Obstet Gynecol. 1977;129(3):260-266.

44. Rose CH, Thigpen B, Bofill JA, et al. Obstetric implications of antepartum corticosteroid therapy for HELLP syndrome. Obstet Gynecol. 2004;104(5 Pt 1):1011-1014.

45. Martin JN, Rose CH, Briery CM. Understanding and managing HELLP syndrome: The integral role of aggressive glucorticoids for mother and child. Am J Obstet Gynecol. 2006;195(4):914-934.

46. Matthys LA, Coppage KH, Lambers DS, et al. Delayed postpartum preeclampsia: an experience of 151 cases. Am J Obstet Gynecol. 2004;190(5):1464-1466.

47. Knight M: on behalf of UKOSS. Eclampsia in the United Kingdom 2005. BJOG. 2007;114(9):1072-1078.

48. Roth C, Ferbert A. The posterior reversible encephalopathy syndrome: what's certain, what's new? Pract Neurol. 2011;11(3):136-144.

49. Munro PT. Management of eclampsia in the accident and emergency department. J Accid Emerg Med. 2000;17(1):7-11.

50. Katz V, Farmer R, Kuller JA. Preeclampsia into eclampsia: Toward a new paradigm. Am J Obst Gynecol. 2000;182(6):1389-1396.

51. Vigil-de Gracia P, Ludmir J. The use of magnesium sulphate for women with severe preeclampsia or eclampsia diagnosed during the postpartum period. J Matern Fetal Neonatal Med. 2015;28(18):22072209 .

52. Aukes AM, De Groot JC, Wiegman MJ, et al. Long-term cerebral imaging after pre-eclampsia. BJOG. 2012;119(9):1117-1122.

53. Aukes AM, de Groot JC, Aarnoudse JG, et al. Brain lesions several years after eclampsia. Am J Obstet Gynecol. 2009;200(5):504.e1-504. e5.

54. Stott VL, Hurrell MA, Anderson TJ. Reversible posterior leukoencephalopathy syndrome: a misnomer reviewed. Intern Med J. 2005;35(2):83-90.

55. Wiegman MJ, Zeeman GG, Aukes AM, et al. Regional distribution of cerebral white matter lesions years after preeclampsia and eclampsia. Obst Gynecol. 2014;123(4):790-795.

56. Brewer J, Owens MY, Wallace K, et al. Posterior reversible encephalopathy syndrome in 46 of 47 patients with eclampsia. Am J Obstet Gynecol. 2013;208(6):468.e1-468.e6.

57. Hefzy HM, Bartynski WS, Boardman JF, et al. Hemorrhage in posterior reversible encephalopathy syndrome: imaging and clinical features. AJNR. Am J Neuroradiol. 2000;30(7):1371-1379. 